\title{
https://doi.org/10.24297/ijct.v20i.8756
}

\section{Design and Implement Beacon based scheme for Node Localization in Underwater Acoustic Network}

\author{
Puneetpal Kaur ${ }^{1}$, Mohit Marwaha², Baljinder Singh $^{3}$ \\ ${ }^{1}$ Research Scholar (M.Tech, Punjab Technical University, India) \\ ${ }^{2}$ Assistant Professor (Beant College of Engg: \& Tech, Punjab Technical University, India) \\ ${ }^{3}$ Assistant Professor (Beant College of Engg: \& Tech, Punjab Technical University, India)
}

\begin{abstract}
A network that can sense the surroundings and collected all the information from the sensor nodes and passed it to the base station is known as a wireless sensor network. The underwater acoustic networks are the type of network deployed under the oceans and passed information to the base station. Due to the dynamic nature of the network, nodes change their location at any time. To maximum aggregate information from the sensor nodes, to estimate exact node location is very important. The sensor node position estimation is a major issue of the underwater acoustic network. The process of estimating node position is called node localization. In the existing RSSI based approach for the node, localization has a high delay, which reduces its efficiency. The technique needs to be designed, which localizes more nodes in less amount of time. This research is based on the advancement of the range based scheme for node localization. In the proposed scheme, mobile beacons are responsible for node localization. The beacon nodes send beacon messages in the network, and sensor nodes respond back with a reply message. When two beacons receive the reply of a sensor node that is considered as a localized node, the sensor nodes which are already localized will not respond back to the beacon messages, which reduce delay in the network for node localization.
\end{abstract}

Keywords: Underwater Acoustic Network, Beacons, Localization.

\section{Introduction}

For collecting information from applications spread across large areas several sensor nodes are deployed and the network generated due to the wireless communication of these nodes is referred as WSN. The data that is collected from surrounding areas is passed on by the sensors to a base station such that further processing can be performed on it. However, the size of a sensor node is very small due to which it includes very less power, memory and also its capacity of perform operations is less. In order to ensure that the complete region is deployed with sensor nodes, random deployment of nodes is done in these networks. This helps in gathering the information from all parts of the region and sending it to the base station. The base station is placed at the center of the network such that the data can be aggregated easily by all the nodes to it [1].

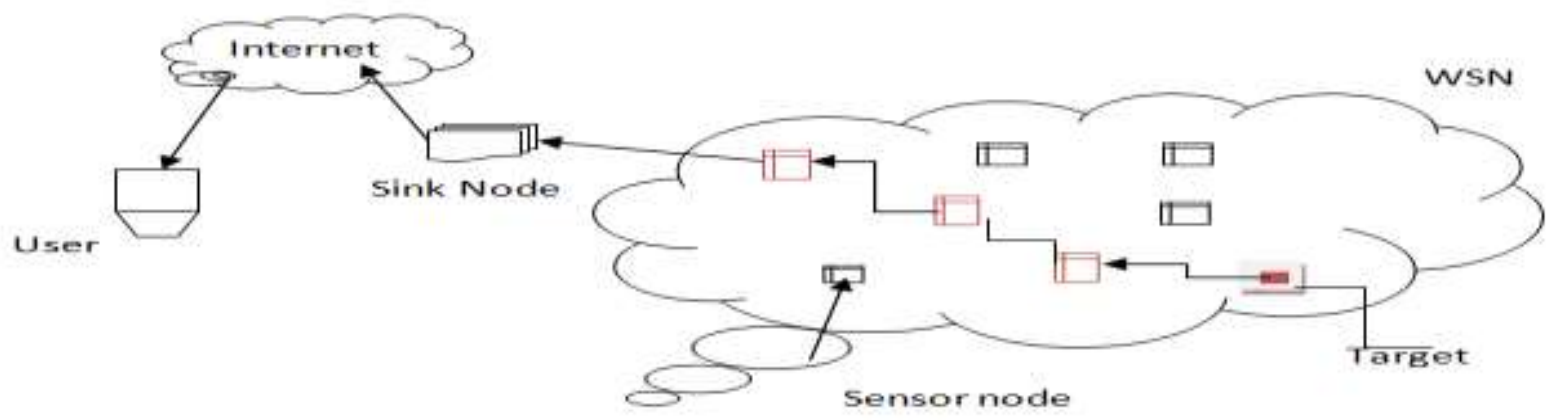

Fig.1.1: Traditional Wireless Sensor Network 
The sensor nodes that are deployed within the network are responsible for monitoring the environmental conditions that exist around the region. All the information is collectively available in the base station. The base stations have higher power for performing operations as well as higher storage capacities. Thus, such type of information can be processed in minimal time by them. A gateway is also present within these networks, which acts as an interface in between the internal as well as external scenarios of the networks [2].

It is possible to access the data that is being received from the base station and further pass it to the end users or clients. Further, this information is also passed on the servers for storage purposes. The deployment of WSNs is done in huge areas. Thus, there are large numbers of base stations deployed within these networks in order to ensure that the huge amount of data that is being gathered by the nodes can be passed ahead at one single instant of time.

WSNs support two different types of communications which are uni-direction as well as bi-directional. The sensor nodes forward the information collected, to the base station and the bidirectional communication mode is used here through which the information is again transmitted back to sensor nodes. The WSNs are deployed by the applications in which it is impossible to provide human surveillance such that the surroundings can be monitored [3]. These networks have their own characteristic features such as the nodes deployed within them are very small in size. The nature of these networks is heterogeneous and the lifetime of these networks is very small. There is very less mobility amongst the sensor nodes and the flat-grid based topology is involved. WSNs are deployed within several applications like military areas or health institutes. The sensor nodes deployed over areas are responsible to gather all the information of surroundings such as the temperature, pressure etc. Any kinds of possible emergency conditions or disasters are also known through the information gathered by sensor nodes.

\section{Underwater acoustic network}

In order to ensure that the important information can be transmitted across the areas and communication can be done amongst users, underwater deployment of networks is done. However, there are certain factors that make it difficult to perform high-speed communications within the acoustic channels like the bandwidth available is limited here, the multi-path and fading are higher as well as the time-variations and the Doppler shifts of water. There is poor propagation of electromagnetic waved in the sea waters. The communication approaches are designed mainly for deploying the terrestrial wired as well as wireless channels. These techniques are to be modified such that appropriate underwater channels can be generated here [13].

Upon the underwater acoustic networks, various researches have been made over the time. Initially, underwater phones were generated for the US navy during the World War II so that the armies could access them in the oceans for communication. Over the time, the communication has been improved by introducing these networks within other scientific, military as well as the commercial applications as well. In the underwater acoustic networks, the mode of communication can be done by using the electromagnetic waves, optical waves or the acoustic waves. There are advantages as well as disadvantages for both of these approaches. For instance, the radio waves are highly affected by the high attenuation of the water which thus results in increasing the transmission power and also requires larger antennas [9]. The optical waves will be affected largely by scattering as well as absorption, even though the data rate communication used by them is higher. Therefore, highly efficient and reliable optical waves can only be provided with the help of short distance links available [14]. B using acoustic waves for communication, one can transmit the information over long distances in comparison to other networks. However, the affects that are caused due to the absorption of wader water is less. So, reliable results are provided with the deployment of these networks. In comparison to other networks, the demand and popularity of these networks is growing day by day and thus the research within them is also expanding. 


\section{Challenges in underwater acoustic sensor networks:}

During the designing of underwater acoustic networks, following are some of the challenges being faced:

1. This approach provides limited bandwidth only.

2. The underwater channel is impaired because of the multi-path provided along with fading.

3. Five degree of orders of propagation delay is identified in this approach in comparison to the Radio Frequency (RF) terrestrial channels.

4. The connectivity here is identified to be of temporary loss and also the bit error rates are higher here [17].

\section{Various Issues in UAWSN:}

i. Path loss: There are several sub-issues identified in this categorization amongst which one is given below [18]:

Attenuation: Attenuation is caused mainly in case when the heat is generated through the transformation of acoustic energy using absorption mechanism. There is increase in the value of attenuation as the distance and frequency is also increased. Scattering, reverberation and various other such factors are responsible for the cause of attenuation in the networks. The value of depth if water is an important factor to be considered in this effect.

\section{ii. Doppler spread:}

The availability of Doppler frequency spread is important when the UW-A channels are present. This also results in degradation of the performance of digital communications. Since the receiver provides higher transmission of data rate in the adjacent symbols, this results in causing interference. There is a need of proper signal processing using the generated ISI.

The Doppler spreading is used to introduce the simple frequency translation that helps in easily compensating the receiver. It is however difficult to spread the frequencies continuously which also includes non-shifter signal [21].

In case when the Doppler spread is caused for a channel, the generation of BT uncorrelated samples are done approximately. The bandwidth $B$ is used by the channel and the time duration $T$ is involved by symbol. When there is very less BT value in comparison to unity, the spreading of channel is done. The Doppler fading results in causing ignorance of effects. However, when BT is higher than unity, overspread is caused here.

\section{iii. Energy conservation:}

Power is needed by the sensor nodes to perform various operations. Some of amount of energy consumption is done for activities such as collection of data, processing it and providing communication. In order to perform various operations, continuous monitoring of the medium is required. Even though no task is being performed at similar time duration, higher amount of energy is being consumed by the nodes and its components. The replacement of power of batteries is not required after the consumption of complete power. However, due to the demographic conditions of the sensors deployed in the network, it is almost impossible to recharge or replace the batteries within the networks [22]. There are several challenges being faced during the design and development of hardware and software protocols that are highly energy efficient. . 


\section{Problem Formulation}

A WSN (Wireless Sensor Network) can be described as a network that sense and collect information about environmental parameters and send this information to the sink (base station). This network faces several challenges related to data aggregation, energy consumption, node localization, and security. This work is focused on the UANs (Underwater Acoustic Networks). This network belongs to that category of SNs (Sensor Networks) using which the underwater conditions are sensed and the sensed information is transmitted to the sink. These networks use tiny sensor devices for sensing the conditions and face a critical issue of energy consumption. It is essential to resolve this issue in proper time for maintaining the efficiency of the network. The sensor devices deployed in these networks have the ability to change their place because of water waves. It is not easy to determine the place of the sensor device. The base paper presents an approach based on beacons. This approach implements beacons that transmit beacon signals and the nodes or devices receiving these signals revert back by sending the ping message. A node becomes local node when the two beacons get respond message from a single node. The approach used in base paper combines angle and distance method for the localization of node. For node localization, the too much intricacy of this approach increases the network delay. The complexity of the algorithm is high because every node need to respond back which is already localized. This increases delay and bandwidth consumption of the network. This work presents an improved version of the existing approach for decreasing delay.

\subsection{Objectives}

1. To study beacon based node localization approach in under water acoustic networks

2. To present improvement in beacon based technique which makes use of threshold approach for node localization.

3. Implement proposed improvement for the node localization in UANs (Underwater Acoustic Networks).

4. To Analyze performance of proposed and existing algorithm in terms of various parameters

- Percentage of nodes localized

- Delay

- Fault detection rate

\section{Research Methodology}

The sensor devices are distributed randomly for deploying an USN (Underwater Sensor Network). The deployment of sensor devices is carried out at different level of deepness for the monitoring of a particular area. In this situation, fixed communication range is provided to the particular sensors by assigning particular monitoring chores. The presented localization algorithm requires the knowledge about the location of some particular nodes beforehand for localizing the other sensor nodes or devices referred as anchor nodes [24]. Some mobile nodes are deployed inside the anchor nodes. These nodes are called as mobile beacons. The mobile nodes can vertically dive and go up on the sea surface using more weight. The mobile nodes go up for reaching the sea surface once they touch the deepest installation point. The node receives their coordinates using GPS receiver once they start to float over the sea surface. Next, the nodes dive into the water in the direction of the monitoring zone. The node reaching to the depth does changes merely in the $z$-coordinate of the mobile beacon. Both mobile beacons and sensor nodes are fitted with a pressure sensor for deepness measurement. For all the mobile beacons, the diving speed and communication range are limited to some level into the water. The nodes diving into the sea make use of acoustic transceiver for broadcasting the localization messages. These nodes send the localization messages at fixed beacon interval (bi). The beacon interval can be described as a fixed time interval in which mobile nodes send the messages. The localization 
messages contain a mobile beacon ID, message number and the coordinates of mobile beacon during instance time. The sensor devices listen to the broadcast messages sent by mobile beacons in passive manner. These messages are used for measuring the distance between the sensor nodes and the mobile beacon [2528]. These networks make assumption regarding the sensor nodes being static. Their remoteness from three mobile beacons is measured for detecting the sensor devices' position. The earlier knowledge about the deepness of every sensor device generates the need to detect the coordinates of $x$ and $y$. The range of monitoring zone can be identified based on the communication range and the beacon interval of mobile beacon [26]. The mobile nodes should be covered largely to ensuring the covering of maximal amount of sensor devices.

Receive Coordinates by GPS
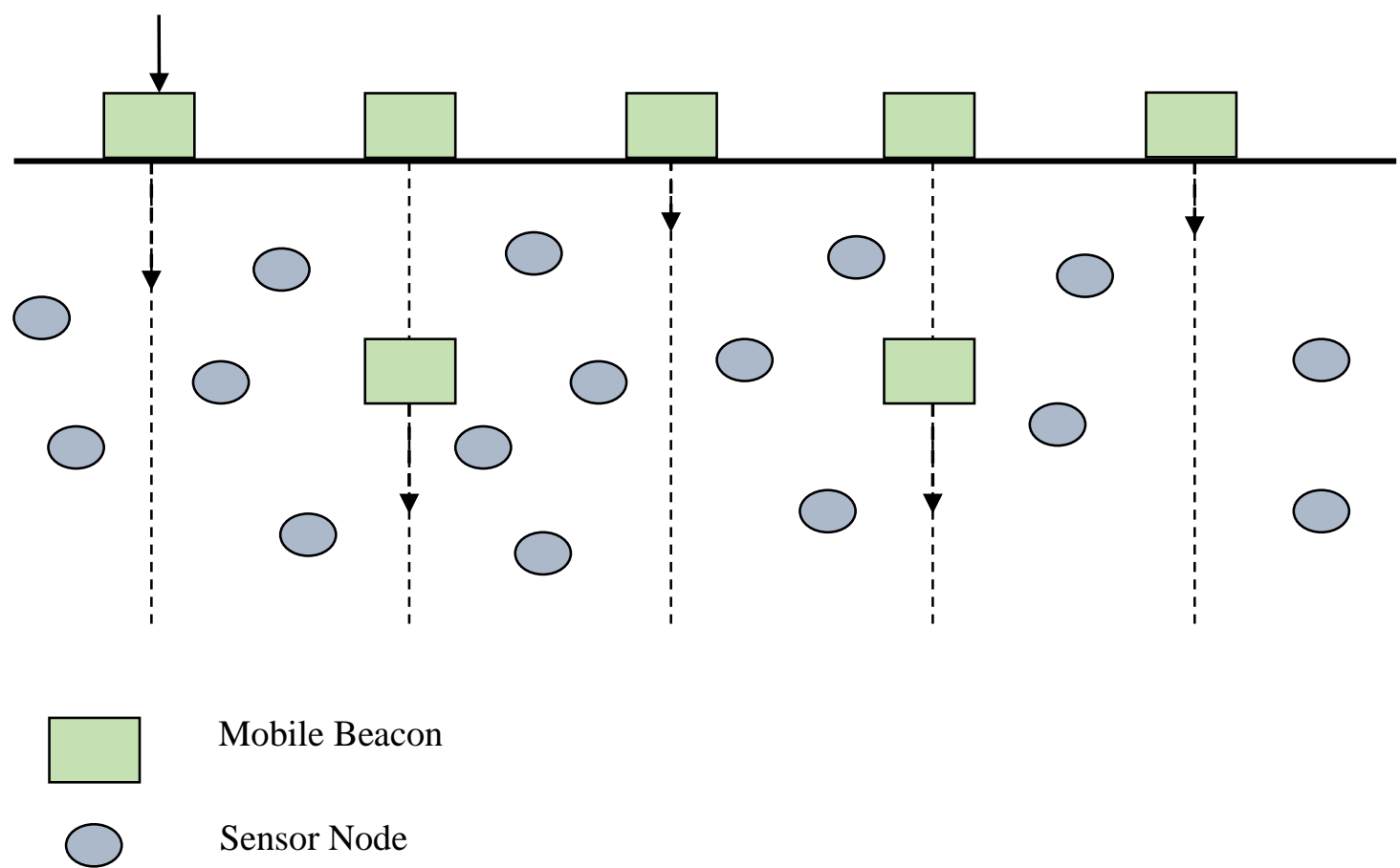

Fig 1.2 Node localization Scenario

For node localization, the advance range based algorithm is the upgrading of the range based algorithm. The new improved algorithm just considers distance metric to localize the node. Figure 3.1 shows the deployment of beacons for localizing the location of sensor device. The beacons broadcasts message messages in the network while sensor devices revert back with the reply messages after receiving these messages [27]. The beacons after receiving reply messages go on to check the time interval. Beacons measure the distance based on the time interval. A sensor node becomes a localized node if the two beacons get respond from this node simultaneously. Beacon shares information regarding distance for finding the node' coordinates [28]. The color of localized node gets change when the beacon transmits the control message to the localized node. The list of nodes localized by a beacon will be forwarded to the other beacon. The other beacon also implements the same process of beacon message transferring across the network. The nodes localized beforehand will not replay back to the mobile nodes. The repetition of the described process is carried out following by the defined amount of time. Once the threshold time is over, all sensor nodes carrying the localized flag will leave this flag. These nodes will be labeled as non-localized nodes [29-30]. In the network, the clock should be synchronized for node localization in the advance range based approach. 


\section{Result and Discussion}

This work implements MATLAB tool for performing evaluations on the new approach. The underwater network is deployed with approximate 250 sensor nodes along with some static nodes. The sound speed of $1500 \mathrm{~m} / \mathrm{s}$ is transmitted in this network at constant rate.

Beacon interval (bi) refers to the transmission range based on which the sensor devices vary in the range from 20 to $250 \mathrm{~m}$. The deployment of anchor nodes is carried out within the network. Also, some more weights are attached with these nodes for fixing their positions. The anchor nodes are facilitated with GPS using which the correct location of these nodes can be found out. The performance analysis of presented approach is carried out by considering several metrics. These metrics include coverage, beacon interval and localization ratio etc. This work presents improvement in the range based approach to localize the node. In the earlier range based approach, the sensor nodes become localized when the beacons broadcast the beacon message across the network and node obtain reply message from the sensor devices. In the new scheme, the beacons show no mobility within the network. Here, a localized sensor node does not reply back after receiving beacon message. Once the anchor nodes broadcast beacon message to the sensor devices, the sensor nodes respond back to the anchor nodes. This results in the solving of linear location to estimate the location of sensor device. The time interval approach is implemented for the measurement of nodes' position. This algorithm uses following formula for the distance estimation.

$\mathrm{d}=\mathrm{v} \times \mathrm{t}$

In the above formula, variable $d$ signifies distance; $v$ denotes the signal speed and $t$ represents time consumed by the signal to cover the distance $d$. hence, it is possible to measure the distance along with the position of the dumb node by measuring the arrival time and the speed of signal propagation. The time taken by a signal to travel from the beacon node to the dumb node is referred as the arrival time. The clocks of sensor nodes should be synchronized for measuring the distance amid the anchor nodes and sensor nodes. The sensor nodes transmit data along with the time of event occurrence. The time lay approach is used for synchronizing the clock after gathering this information [20]. The beacon gets reply message and measures distance using Euclidian distance formula. This decreases the network overhead which in turn decreases delay. Inverse distance is computed for estimating the sensor node's coordinates. Here, $(x, y)$ denotes the location for unidentified node $D$. Also, $\left(x_{i}, y_{i}\right)$ is assumed as known location for $i$ 'th anchor node receiver. Allow $d_{i}$ be the i'th anchor node for unidentified nodes,. Further, allow $\mathrm{n}$ be the total number of anchors fixed across the network. Following formula is used to measure the location in range free localization:

$$
\left\{\begin{array}{c}
\sqrt{\left(x-x_{1}\right)^{2}+\left(y-y_{1}\right)^{2}}=d_{1} \\
\sqrt{\left(x-x_{2}\right)^{2}+\left(y-y_{2}\right)^{2}}=d_{2} \\
\vdots \\
\sqrt{\left(x-x_{i}\right)^{2}+\left(y-y_{i}\right)^{2}}=d_{i}
\end{array}\right.
$$

$$
A=-2 \times\left(\begin{array}{c} 
\\
x_{1}-x_{n} y_{1}-y_{n} \\
x_{2}-x_{n} y_{2}-y_{n} \\
\vdots \\
\vdots \\
\vdots \\
x_{m-1}-x_{m} y_{m}-1-y_{n}
\end{array}\right)
$$

$B=\left(\begin{array}{c}d_{1}^{2}-d_{n}^{2}-x_{1}^{2}+x_{n}^{2}-y_{1}^{2}+y_{n}^{2} \\ d_{2}^{2}-d_{n}^{2}-x_{2}^{2}+x_{n}^{2}-y_{2}^{2}+y_{n}^{2} \\ \vdots \\ \cdot \\ \cdot \\ d_{n-1}^{2}-d_{n}^{2}-x_{n-1}^{2}+x_{n}^{2}-y_{n-1}^{2}+y_{n}^{2}\end{array}\right)$ 
$P=\left(\begin{array}{l}x \\ y\end{array}\right)$

Here, $P=\left(A^{T} A\right)^{-1} A^{T} B$

In the above formula, P denotes the sensor nodes' coordinates.

a. Coverage: Coverage represents how well or to which extent a sensor node vigilant the every point of an installed network. $\mathrm{R}$ is supposed to be the transmission range for a mobile beacon. Here, $\mathrm{S}_{2}$ denotes the speed of a mobile beacon. This speed remains constant and must be identified previously for each sensor device. $D_{b}$ signifies the distance covered by a mobile beacon ahead of the transmission of message further. Therefore, equation (1) measures the coverage of a mobile beacon as:

Coverage $=\frac{\Pi}{12}\left(4 R+D_{b}\right)\left(2 R-D_{b}\right)^{2}$

b. Localization ratio: This metric signifies the amount of sensor nodes which may get localized.

c. Number of localized nodes: In the equation given below, 'N1' represents the number of nodes which can be localized by a mobile beacon. $N 1$ Can be measured as:

$N 1=\frac{N}{V} * \frac{\Pi}{12}\left(4 R+D_{b}\right)\left(2 R-D_{b}\right)^{2}$

In this equation, $\mathrm{N}$ signifies the total number of nodes in a network while $\mathrm{V}$ represents the volume of the installed nodes.

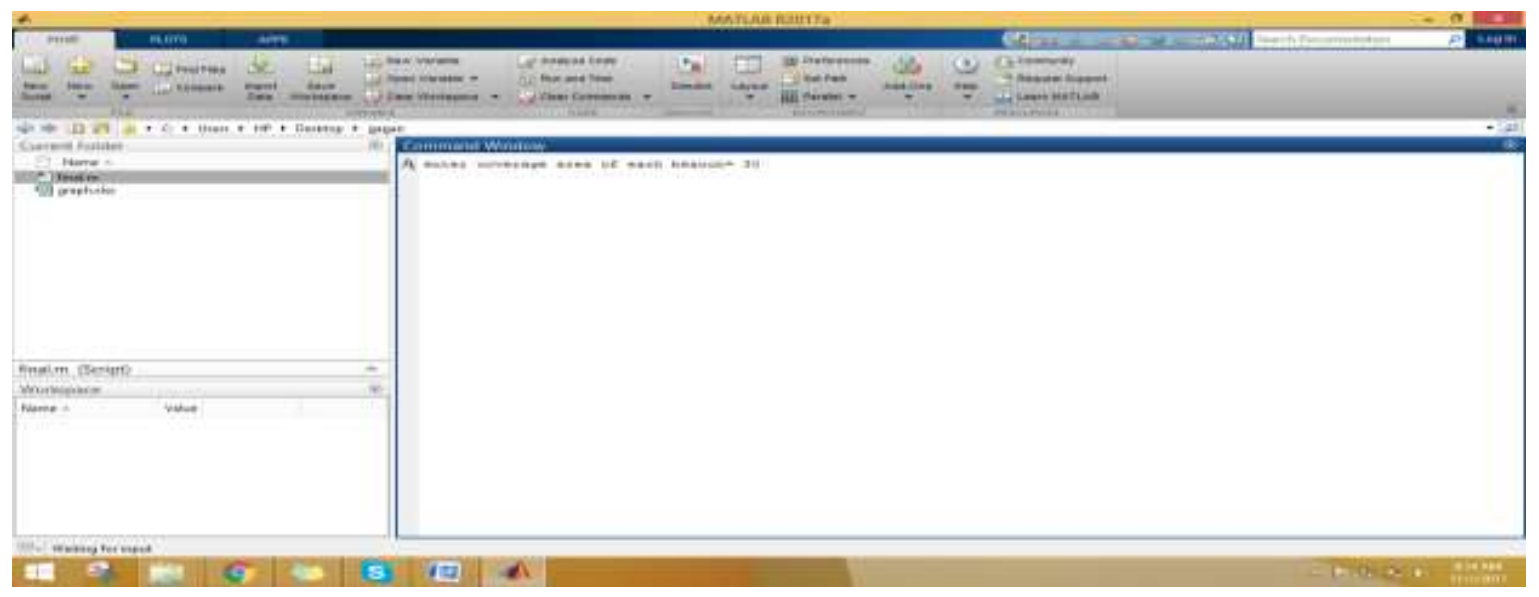

Fig 1.3 Define Beacon coverage Area

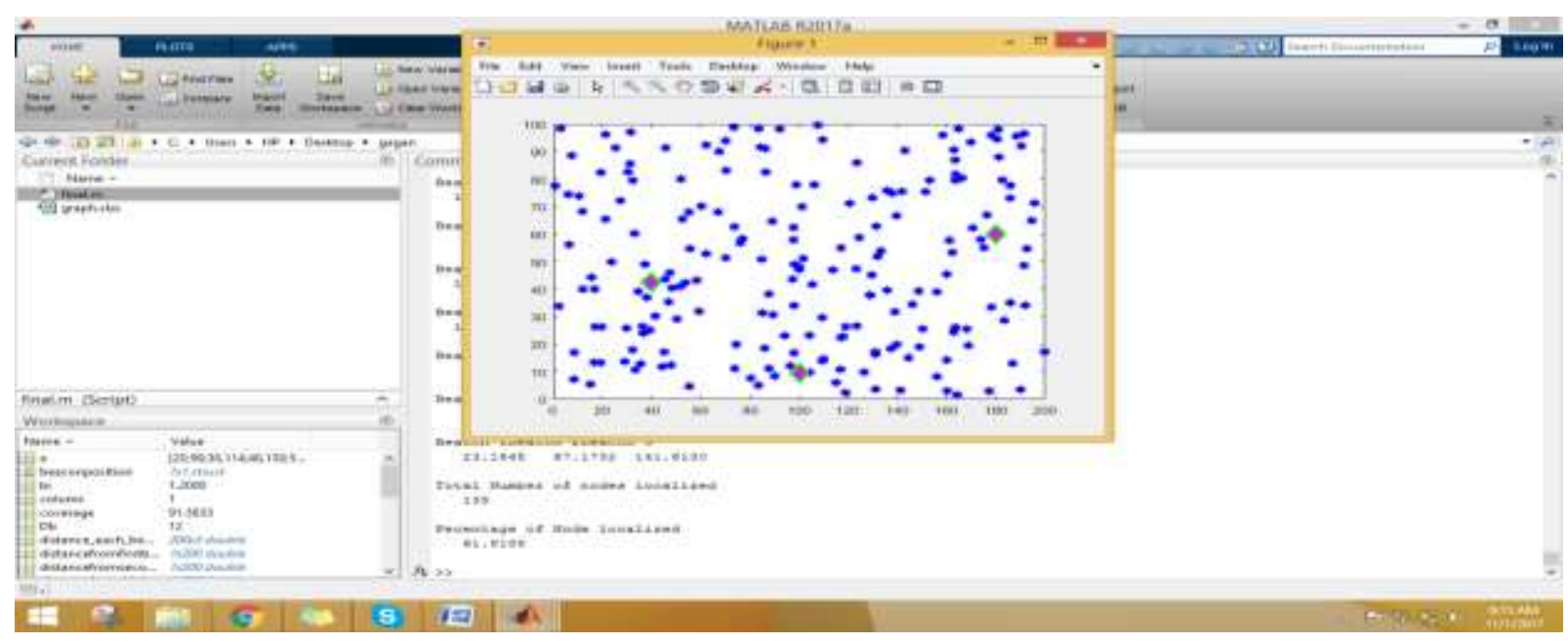

Fig 1.4 Network deployment 
Figure below shows the network installed with fixed number of sensor devices. Beacons are inserted within the network and these beacons can change their location horizontally. The coverage area of the beacons are defined in the last figure which can localize the sensor nodes

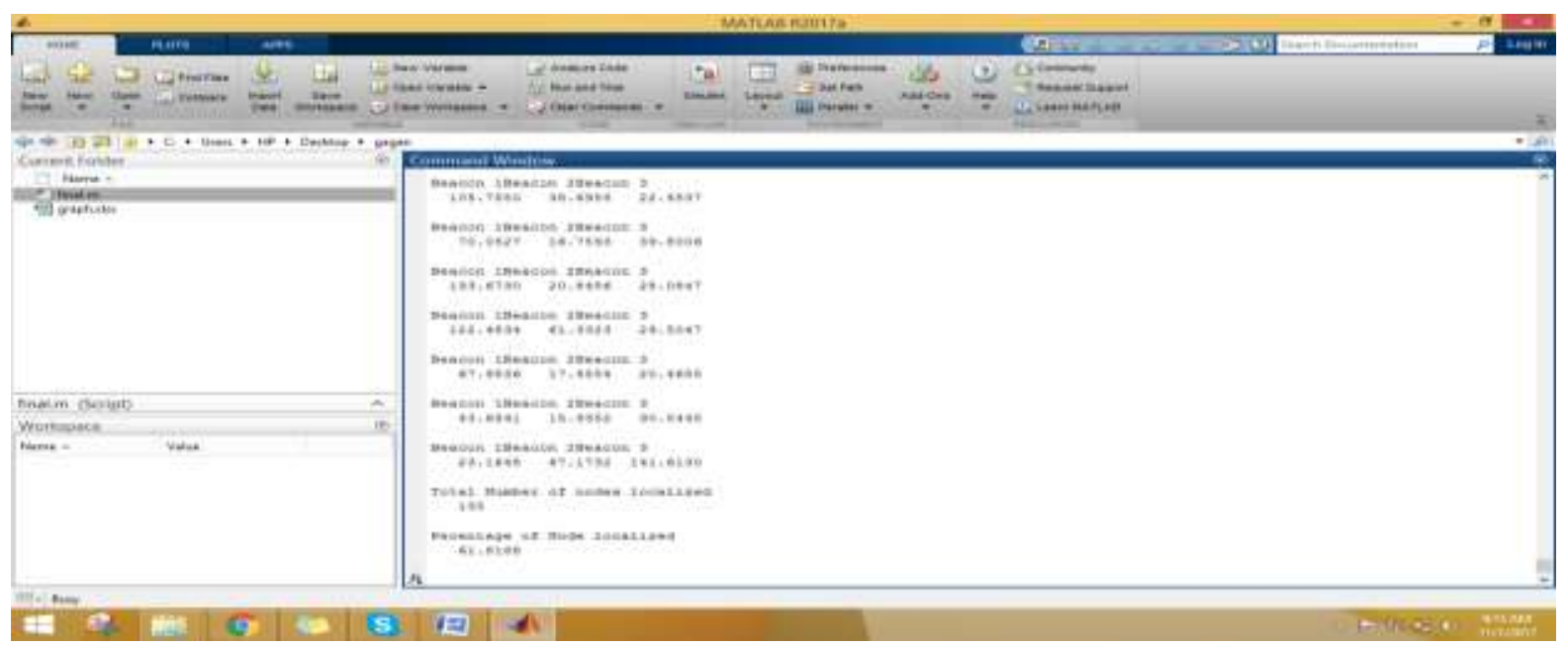

Fig 1.5 Distance of each node from each beacon

Figure above shows the implementation of new algorithm. This algorithm check the distance of beacon from the sensor node. The node which is covered with in the range of two beacons get localized.

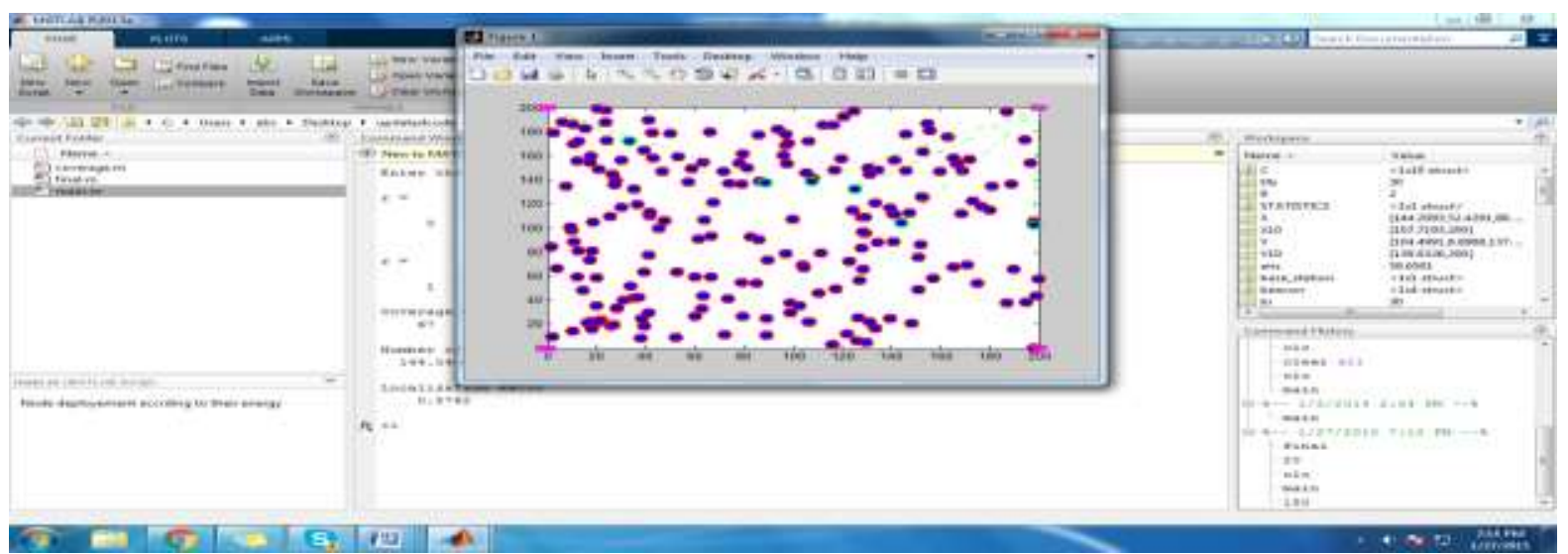

Fig 1.6 Node Localization

Figure above shows that the four beacons are used in the network for the node localization. The beacons transfers the beacon frames across network for localizing the node. The nodes when receive the beacon frame revert back while beacons measure the distance of the sensor node.

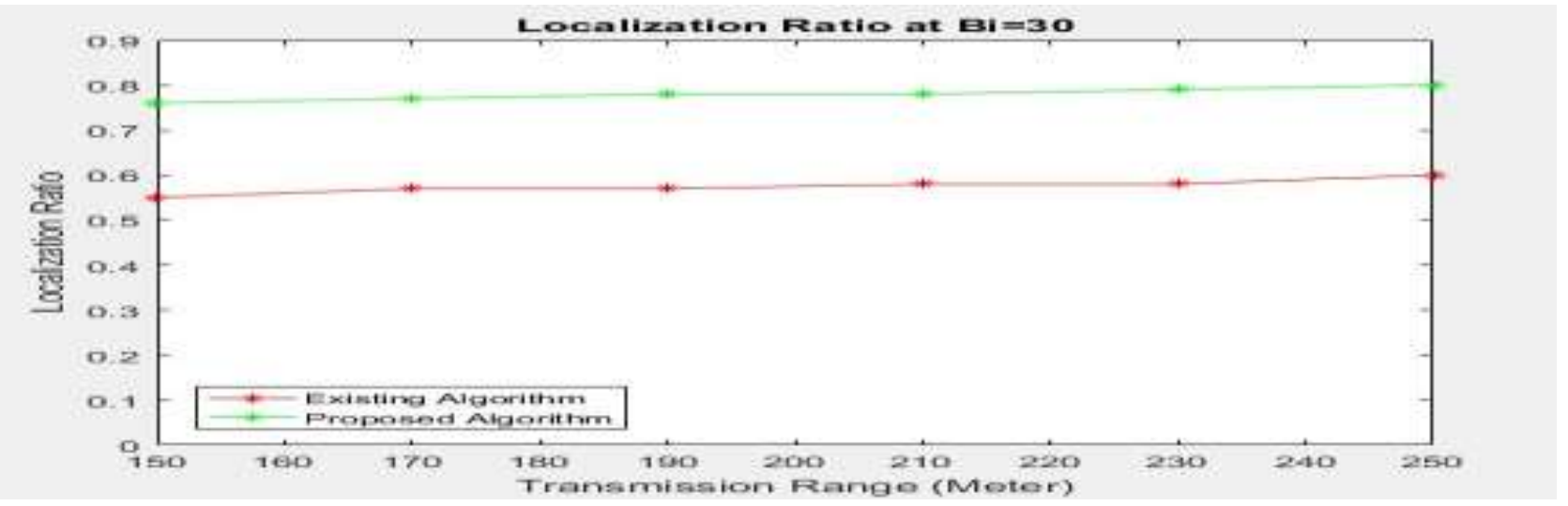

Fig 1.7 Localization Ratio Comparison 
Figure above shows the comparison of the new and the earlier algorithms in terms of localization ratio. In the new approach, the coverage area of beacons is more than the earlier approach. As a result, the localization ratio increases at steady rate.

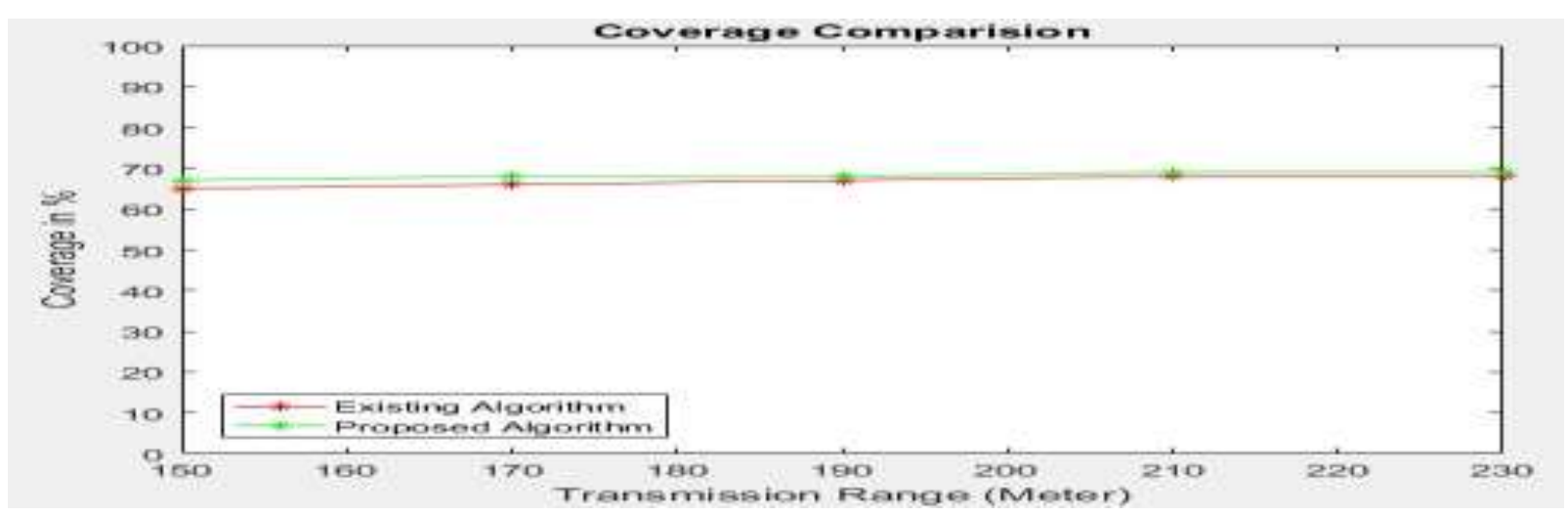

Fig 1.7 Coverage Comparison

Figure below shows the comparison of the new and the earlier algorithms in terms of localization ratio. In contrast to the earlier approach, the presented approach has high coverage area as it uses more beacons. In the new algorithm, the coverage enlarges at constant rate with the increase in transmission range.

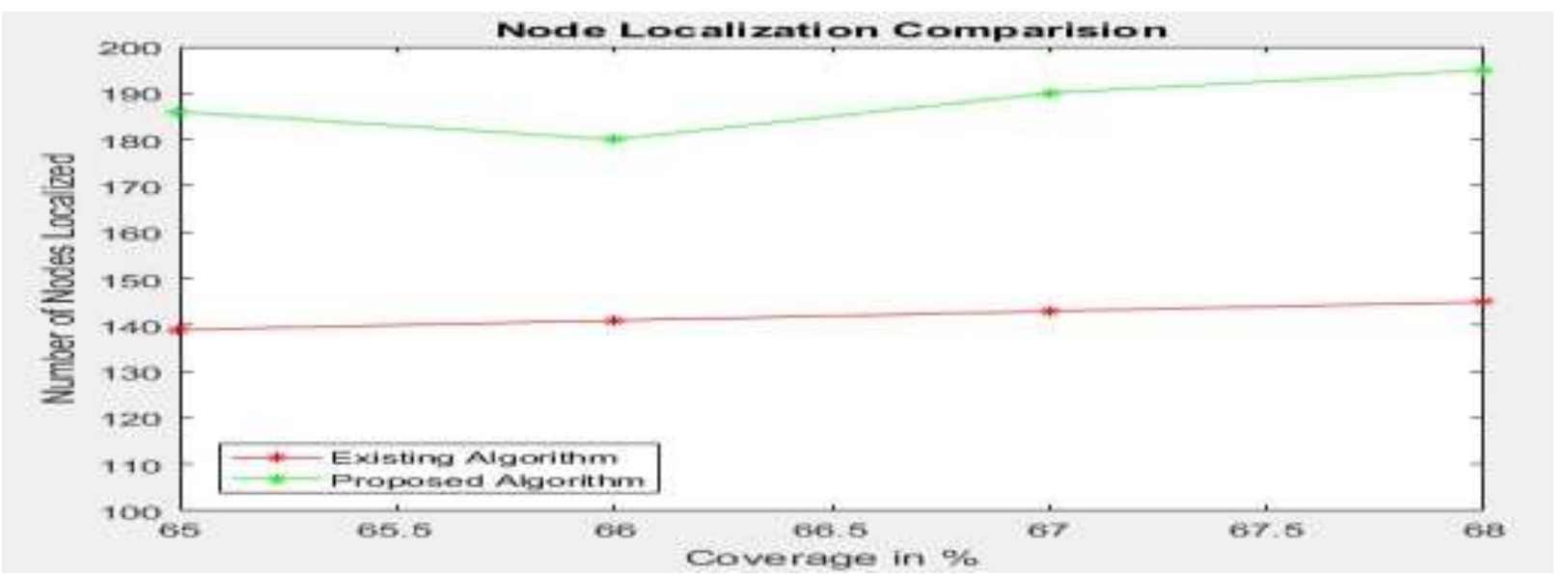

Fig 1.8 Number of Nodes localization Comparison

Figure above shows the comparison of the new and the earlier algorithm based on the number of localized nodes against coverage area. The number of localized nodes enlarges with the coverage area in both of these algorithms. In contrast to the earlier algorithm, the new algorithm has more number of localized nodes.

Table 1: Range and Localization Ratio

\begin{tabular}{|l|l|l|}
\hline Range & $\begin{array}{l}\text { Existing } \\
\text { Algorithm }\end{array}$ & $\begin{array}{l}\text { Proposed } \\
\text { Algorithm }\end{array}$ \\
\hline 150 & 0.57 & 0.77 \\
\hline 170 & 0.57 & 0.78 \\
\hline 190 & 0.58 & 0.78 \\
\hline 230 & 0.58 & 0.79 \\
\hline 250 & 0.60 & 0.80 \\
\hline
\end{tabular}


Table 1 compares the earlier and the presented algorithm in terms of the ratio of localization. In contrast to the earlier algorithm, the new algorithm shows higher node localization while the range increases at the similar rate.

Table 2: Coverage and Number of Nodes Localized

\begin{tabular}{|l|l|l|}
\hline Coverage \% & Existing Algorithm & Proposed Algorithm \\
\hline 65 & 139 & 186 \\
\hline 66 & 141 & 180 \\
\hline 67 & 143 & 190 \\
\hline 68 & 145 & $195 \mathrm{~s}$ \\
\hline
\end{tabular}

Table 2 compares the earlier and the presented algorithm in terms of number of nodes localized vs. coverage area. In contrast to the earlier algorithm, the presented algorithm contains more number of localized nodes

Table 3: Range and Coverage Area

\begin{tabular}{|l|l|l|}
\hline Range $(\mathrm{m})$ & Existing Algorithm & Proposed Algorithm \\
\hline 150 & $65 \%$ & $67 \%$ \\
\hline 170 & $66 \%$ & $68 \%$ \\
\hline 190 & $67 \%$ & $68 \%$ \\
\hline 210 & $68 \%$ & $69 \%$ \\
\hline 230 & $68 \%$ & $69 \%$ \\
\hline
\end{tabular}

Table 3 compares the earlier and the presented algorithm in terms of coverage area. As per the analysis, the new approach has more coverage area in contrast to the earlier approach.

\section{Conclusion \& Future Work}

In under water acoustic networks, the approach used to estimate the location of sensors is known as node localization. This work makes improvement in the existing range based algorithm for node localization. In the new algorithm, mobile beacons broadcast beacon messages across the network and the nodes replying back are labeled as the localized nodes. The localized nodes don't reply back after receiving beacon message from mobile beacons for the second time. This ultimately decreases the localization delay. This also increases the energy efficiency of the network. As per the analysis, the new approach performs the node localization task in lesser time than the earlier approach. This work makes use of MATLAB tool for implementing the new algorithm. The new algorithm performs better than the earlier algorithm in terms of node localization verses coverage area of mobile beacons and node localization ratio. 


\section{Future Work}

The futuristic work would involve these tasks:

1. The new approach can be further improved for the secure node localization schemes.

2. The proposed algorithm can be enhanced using the optimization algorithms for the reduction of MSE value.

3. The comparison of new approach will be carried out against the other efficient node localization approaches.

\section{References}

[1] Gouvy, N., Hamouda, E., Mitton, N., \& Zorbas, D., "Energy efficient multi-flow routing in mobile Sensor Networks", IEEE In Wireless Communications and Networking Conference (WCNC), vol. 3, pp. 1968-1973, 2013.

[2] Gowrishankar.S 1 , T.G.Basavaraju 2, Manjaiah D.H 3 , Subir Kumar Sarkar," Issues in wireless sensor networks," 2008, WCE, vol.1, pp 5-15

[3] M.H. Anisi, A.H. Abdullah, and S.A. Razak, "Energy-Efficient Data Collection in Wireless Sensor Networks", 2011, Wireless Sensor Networks, vol. 3, pp. 329-333.

[4] P. Mohanty, S. Panigrahi, N. Sarma, and S.S. Satapathy, "Security Issues In Wireless Sensor Network Data Gathering Protocols: A Survey", 2005-2010, Journal of Theoretical and Applied Information Technology, vol. 13, no.1, pp. 14-27.

[5] M.K. Jain, "Wireless Sensor Networks: Security Issues and Challenges", 2011, International Journal of Computer and Information Technology, vol. 2, no. 1, pp. 62-67.

[6] A.K. Pathan, "Security in Wireless Sensor Networks: Issues and Challenges", 2006, Proc. 8th International Conf. Advanced Communication Technology, vol. 2, pp. 1043-1050.

[7] Salvador Climent, Antonio Sanchez, Juan Vicente Capella, Nirvana Meratnia and Juan Jose Serrano, "Underwater Acoustic Wireless Sensor Networks: Advances and Future Trends in Physical, MAC and Routing Layers", Sensors, vol.14, pp.795-833, 2014.

[8] Benson, B. Design of a Low-cost Underwater Acoustic Modem for Short-Range Sensor Networking Appications. Ph.D. Thesis, University of California, San Diego, CA, USA, 2010.

[9] J. Heidemann, M. Stojanovic, M. Zorzi, Underwater sensor networks: Applications, advances and challenges. Philos. Trans. R. Soc. A, vol.370, pp.158-175, 2012.

[10] Akyildiz, I.F.; Pompili, D.; Melodia, T. Underwater acoustic sensor networks: Research challenges. Ad. Hoc. Netw. , vol.3, pp.257-279, 2005.

[11] A. Caiti, P. Felisberto, T. Husoy, S.M. Jesusl. Karasalo R. Massimelli, T.A. Reinenk and A. Silva, "UAN Underwater Acoustic Network", IEEE, vol.7, pp.46-53, 2011.

[12] Manjula.R.B, Sunilkumar S. Manvi, "Issues in Underwater Acoustic Sensor Networks", International Journal of Computer and Electrical Engineering, vol.3, pp.101-110, 2011. 
[13] J.H. Cui, J. Kong, M. Gerla, and S. Zhou, Challenges: Building scalable mobile underwater wireless sensor networks for aquatic applications, IEEE Network, Special Issue on Wireless Sensor Networking, pp. 12-18, 2006.

[14] X. Yang, K.G. Ong, W.R. Dreschel, K. Zeng, C.S. Mungle, and C.A. Grimes, "Design of a wireless sensor network for long-term, insitu monitoring of an aqueous environment", vol. 11, pp. 455-472, 2002.

[15] Vasilescu.K.Kotay and D.Rus, Data Collection, Storage and Retrieval with an underwater Sensor Network, ACM SenSys, vol.5 pp.154-165, 2005.

[16] I. F. Akyildiz, D. Pompili, and T. Melodia, Underwater acoustic sensor networks: Research challenges, Ad Hoc Networks, vol.7, pp. 257-279, 2005.

[17] Q. Zhang , and M. Cheng, "A node localization algorithm for wireless sensor network based on improved particle swarm optimization", Lecture Notes in Electrical Engineering, 2014, vol. 237, pp.135-144

[18] Sharma, A., \& Gaffar.H, A., "A Survey on Routing Protocols for Underwater Sensor Networks", 2012, International Journal of Computer Science \& Communication Networks, 74-82

[19] P.K Singh, Bharat Tripathi, Narendra Pal Singh, "NODE LOCALIZATION IN WIRELESS SENSOR NETWORKS," 2011, IJCSIT, vol.2, pp. 2568-2572

[20] Satvir Singh, Shivangna, Etika Mittal, "Range based wireless sensor node localization using PSO and BBO and its variants", International conference on communication systems and network technologies, 2013, vol.4, pp. $9-20$

[21] Avinash Kaur, Sonu Agrawa, "Location detection in wireless sensor network using classical optimization methodology," 2012, IJCST, vol. 3, pp.108-115.

[22] Asma Mesmoudi, Mohammed Feham, Nabila Labraoui, "Wireless sensor networks localization algorithms: a comprehensive survey", 2013, International Journal of Computer Networks \& Communications (IJCNC) vol.5, pp. 112-120. 